\title{
Favoring, Likelihoodism, and Bayesianism
}

\author{
BRANDEN FITELSON \\ Rutgers University
}

In Chapter 1 of Evidence and Evolution, Sober (2008) defends a Likelihodist account of favoring. The main tenet of Likelihoodism is the so-called Law of Likelihood. In this note, I explain why the Law of Likelihood fails to undergird an adequate explication of favoring.

\section{Some Background on the Favoring Relation}

This (brief) note is about the (evidential) "favoring" relation. Pre-theoretically, favoring is a three-place (epistemic) relation, between an evidential proposition $E$ and two hypotheses $H_{1}$ and $\mathrm{H}_{2}$. Favoring relations are expressed via locutions of the form:

$$
\text { E favors } H_{1} \text { over } H_{2}
$$

Strictly speaking, favoring should really be thought of as a four-place relation, between $E, H_{1}, H_{2}$, and a corpus of background evidence $K$. But, for present purposes (which won't address issues involving $K$ ), I will suppress the background corpus, so as to simplify our discussion. Moreover, the favoring relation is meant to be a propositional epistemic relation, as opposed to a doxastic epistemic relation. That is, the favoring relation is not meant to be restricted to bodies of evidence that are possessed (as evidence) by some actual agent(s), or to hypotheses that are (in fact) entertained by some actual agent(s). In this sense, favoring is analogous to the relation of propositional justification - as opposed to doxastic justification (Conee 1980).

In order to facilitate a comparison of Likelihoodist $v s$ Bayesian explications of favoring, I will presuppose the following bridge principle, linking favoring and evidential support:

- E favors $H_{1}$ over $H_{2}$ iff $E$ supports $H_{1}$ more strongly than E supports $H_{2}{ }^{1}$

Finally, I will only be discussing instances of the favoring relation involving contingent, empirical claims. So, it is to be understood that "favoring" will not apply if any of $E, H_{1}$, or $H_{2}$ are non-contingent (and/or non-empirical). With this background in place, we're ready to begin.

\section{A Popperian Sufficient Condition for Favoring}

Here is an eminently plausible sufficient condition for favoring:

$1 \quad$ Likelihoodists may balk at this presupposition. But, as I explain in some detail elsewhere (Fitelson 2007), without this bridge principle, no meaningful comparison between Likelihoodism and Bayesianism seems possible. 
If $H_{2}$ entails $\sim E$ but $H_{1}$ does not entail $\sim E$, then $E$ favors $H_{1}$ over $H_{2}$.

This is a (weak) "Popperian Principle" concerning the evidential asymmetry between refutation and non-refutation. The Popperian slogan for (PP) would be:

- Non-refuting evidence supports more strongly than refuting evidence.

This slogan expresses the kernel of truth in Popperian Falsificationism. The so-called Law of Likelihood (Sober 2008, Royall 1997) is meant to probabilistically generalize (PP). To wit:

Suppose $H_{1}$ confers probability $p_{1}$ on $E$, and $H_{2}$ confers probability $p_{2}$ on $E$. Then, $E$ favors $H_{1}$ over $H_{2}$ iff $p_{1}>p_{2}$.

In other words, (LL) reduces favoring to a comparison of the likelihoods of the $H_{1}$ and $H_{2}$, relative to evidence $E$ [viz., $p_{1}=\operatorname{Pr}\left(E \mid H_{1}\right)$ and $\left.p_{2}=\operatorname{Pr}\left(E \mid H_{2}\right)\right]$. In the limiting, deductive case involved in (PP), $p_{2}=0$ and $p_{1}>0$. And, in such special cases, every (adequate) theory of favoring will endorse the conclusion implied by (LL) [viz., (PP)]. As such, I accept (PP) as a sufficient condition for favoring, and I think (LL) is OK in these special, "Popperian" cases.

However, when we look at the consequences of (LL) for other cases, we can see that it over-generalizes (PP). A useful way to illustrate the nature of (LL)'s over-generalization of (PP) is to consider another, non-Popperian (deductive) sufficient condition for favoring.

\section{A Non-Popperian Sufficient Condition for Favoring}

To see why (LL) over-generalizes (PP), consider another (deductive, limiting case) sufficient condition for favoring that I think should be as uncontroversial as (PP):

If $E$ entails $H_{1}$ and $E$ does not entail $H_{2}$, then $E$ favors $H_{1}$ over $H_{2}$.

Principle $\left(^{*}\right)$ can be thought of as a "dual" of Principle (PP). Basically, $(*)$ is meant to imply that if E conclusively supports $H_{1}$, but $E$ non-conclusively supports $H_{2}$, then $E$ favors $H_{1}$ over $H_{2}$. Consequently, the slogan for $(*)$ would be:

- Conclusive evidence supports more strongly than non-conclusive evidence.

To my mind, this "dual" of (PP) seems just as plausible as (PP) itself. ${ }^{2}$ But, while (PP) is (severally) compatible with each of (LL) and (*), it turns out that (LL) is incompatible with principle $(*)$. Here is a concrete example illustrating the incompatibility of (LL) and $(*)$.

\footnotetext{
2 If one is a Popperian Falsificationist - in a strong, Critical Rationalist sense (Miller 1994) - then one will deny (*). But, that version of Falsificationism is false. And, I take it that contemporary defenders of (LL) [e.g., Sober (2008) and Royall (1997)] do not want to embrace this stronger (and highly implausible) Popperian position. As such, contemporary Likelihoodists will need a different way to argue that (LL) does not over-generalize (PP).
} 
Example. Suppose we have deck of 100 playing cards, and we know nothing about how the cards in the deck are distributed, except for the following two facts: (i) there are some clubs and some red cards in the deck, and (ii) at least one ace of spades is contained in the deck. We shuffle the cards well, and we sample a card (c) at random. Now, consider the following three claims regarding $c$ :

(E) $\quad c$ is a spade.

$\left(H_{1}\right) \quad c$ is a black card.

$\left(H_{2}\right) \quad c$ is an ace of spades.

Because $E$ entails $H_{1}$ and $E$ does not entail $H_{2},(*)$ implies that $E$ favors $H_{1}$ over $H_{2}$ in this case (which clearly seems to be the correct verdict). However, because $\operatorname{Pr}\left(E \mid H_{2}\right)=1>\operatorname{Pr}\left(E \mid H_{1}\right)>0$, (LL) implies that $E$ favors $H_{2}$ over $H_{1}$, which contradicts ( $*$ ). This shows that, while (LL) can be seen as generalizing one sufficient condition for favoring [(PP)], it also contradicts another sufficient condition for favoring $[(*)] .^{3}$

\section{Bayesian Diagnoses (and Explications)}

From a Bayesian point of view, the debate about (LL) is really just a debate about the proper measure of degree of confirmation. Recall our bridge principle connecting favoring and support:

- E favors $H_{1}$ over $H_{2}$ iff $E$ supports $H_{1}$ more strongly than $E$ supports $H_{2}$.

Bayesian confirmation theory provides various explications of "the degree to which $E$ supports $H$." These come in the form of various relevance measures $\mathrm{c}(H, E)$ of "the degree to E confirms $H$. ." For each of these precise Bayesian explications of evidential support, we get a precise confirmation-theoretic bridge principle, of the following kind:

$$
\text { E favors } \mathrm{H}_{1} \text { over } \mathrm{H}_{2} \text { - according to measure } \mathrm{c}-\text { iff } \mathrm{c}\left(H_{1}, E\right)>\mathrm{c}\left(H_{2}, E\right) \text {. }
$$

Different choices of $\mathrm{c}$ lead to different precise Bayesian bridge principles connecting favoring and confirmation. For instance, according to the ratio measure of degree of confirmation:

$$
\text { The degree to which } E \text { confirms } H=r(H, E)=\operatorname{Pr}(H \mid E) / \operatorname{Pr}(H) \text {. }
$$

\footnotetext{
3 The entailment relations are inessential to the intuitive verdicts here. A simple modification of Example drives this point home. Suppose that a highly (but imperfectly) reliable witness is going to make three claims about $c$. The witness is going to report (1) either $E$ or $\sim E$, and (2) either $H_{1}$ or $\sim H_{1}$, and (3) either $H_{2}$ or $\sim H_{2}$. Now, preface each of $(E),\left(H_{1}\right)$, and $\left(H_{2}\right)$ with the following: "The highly (but imperfectly) reliable witness testified that...". This modification does not undermine the intuitive verdict that $E$ favors $H_{1}$ over $H_{2}$ in the Example. Moreover, (LL) will (still) give the (intuitively) incorrect verdict here. And, this is despite the fact that there are no entailment relations between the propositions in the revised testimonial rendition of the Example. So, the entailments are inessential to the Example. See (Fitelson 2007) and (Fitelson 2011) for more detailed diagnoses and discussions.
} 
If we accept $(r)$, then (LL) follows from the resulting bridge principle $\left(\mathrm{BP}_{r}\right)$. That is, if we plug $c(H, E)=r(H, E)$ into $\left(\mathrm{BP}_{\mathrm{c}}\right)$, we get $(\mathrm{LL})$. This allows us to see that $(\mathrm{LL})$ is just a consequence of one approach to Bayesian confirmation.

Unfortunately, the ratio-measure approach to Bayesian confirmation $(r)$ is flawed in various ways. Perhaps the most telling objection to $(r)$ is that it entails commutativity of "degree of evidential support" (Eells \& Fitelson 2002):

$$
\text { For all } E \text { and } H, \mathrm{c}(H, E)=\mathrm{c}(E, H) \text {. }
$$

But, (C) is clearly incorrect, since (e.g.) $E$ might entail $H$, while $H$ does not entail $E$. And, in such cases, it is clear that commutativity of evidential support (hence, degree of confirmation) can fail. I think this flaw is one of the underlying reasons why (LL) gives counter-intuitive results, including those which contradict the intuitively compelling sufficient condition for favoring articulated by principle $(*)$.

There are various (Bayesian) alternatives to (LL)/(r) that are compatible with both (PP) and $(*)$, and which do not imply the commutativity of quantitative confirmation. One naïve Bayesian alternative to (LL) would involve a comparison of posteriors $\operatorname{Pr}\left(H_{1} \mid E\right)$ and $\operatorname{Pr}\left(H_{2} \mid E\right)$ :

$$
E \text { favors } H_{1} \text { over } H_{2} \text { iff } \operatorname{Pr}\left(H_{1} \mid E\right)>\operatorname{Pr}\left(H_{2} \mid E\right) \text {. }
$$

But, this "naïve Bayes" approach to favoring (NB) is also inadequate. Popper (1954) showed that (NB) violates the following sensitivity to evidential relevance requirement:

Suppose $E$ is positively relevant to $H_{1}$ and $E$ is negatively relevant to $H_{2}$. Then, $E$ does not favor $\mathrm{H}_{2}$ over $H_{1}$.

Principle (R) makes sense because favoring is a relation of comparative evidential support. Moreover, (LL) entails (R), so (R) is something that Likelihoodists must (also) accept. In this sense, (R) is common ground between Likelihoodists and Bayesians. That is, anyone who accepts any version of $\left(\mathrm{BP}_{\mathrm{c}}\right)$ must also accept $(\mathrm{R})$. This covers all Bayesian explications of favoring including the Likelihoodist $\left[v i z .,\left(\mathrm{BP}_{r}\right) /(\mathrm{LL})\right]$ approach.

To sum up: we seek an (probabilistic) explication of favoring that is compatible with (PP), $(*)$, and $(\mathrm{R})$. As it happens, there are many such contenders within the Bayesian stable.

At the quantitative level, there are various measures of confirmation (c) that undergird via $\left(\mathrm{BP}_{\mathrm{c}}\right)$ - explications of favoring that are compatible with $(\mathrm{PP}),(*)$, and $(\mathrm{R})$. For instance,

- Likelihood-ratio-based measures (Good 1984, Fitelson 2007, Fitelson 2011).

- An alternative to the likelihood-ratio, which has recently been defended by some philosophers and cognitive scientists (Crupi et. al. 2007, Tentori et. al. 2007).

At the qualitative level, there are various sets of probabilistic sufficient conditions for favoring that can be seen as (proper) generalizations of (PP), $(*)$, and (R). For instance, 
(WLL) Suppose $\operatorname{Pr}\left(E \mid H_{1}\right)>\operatorname{Pr}\left(E \mid H_{2}\right)$ and $\operatorname{Pr}\left(E \mid \sim H_{1}\right) \leq \operatorname{Pr}\left(E \mid \sim H_{2}\right)$.

Then, $E$ favors $H_{1}$ over $H_{2}$.

Joyce (2008) calls this the "Weak Law of Likelihood" [aptly, since (LL) entails (WLL), but not conversely]. It's a principle that (almost all) Bayesian approaches to favoring [based on $\left(\mathrm{BP}_{\mathrm{c}}\right)$ ] will agree upon. Of course, (WLL) appeals to so-called "catch-all likelihoods" $\left[\right.$ viz., $\operatorname{Pr}\left(E \mid \sim H_{1}\right)$ and $\operatorname{Pr}\left(E \mid \sim H_{2}\right)$ ], and so it(s antecendent) will be controversial for many non-Bayesian philosophers. I don't have the space here to delve into these more subtle aspects of the dialectic between Likelihoodism and its Bayesian rivals. For a detailed discussion of the Likelihoodism/Bayesianism debate about the favoring relation, see (Fitelson 2007). And, for a more recent discussion of "constrastivism", Likelihoodism, and Bayesianism — with applications to problems in philosophy of science and cognitive science - see (Fitelson 2011).

\section{References}

Conee, E. 1980. "Propositional Justification”. Philosophical Studies 38: 65-68.

Crupi, V., Tentori, K. and Gonzalez, M. 2007. "On Bayesian measures of evidential support: Theoretical and empirical issues”. Philosophy of Science 74: 229-252.

Eells, E. and Fitelson, B. 2002. "Symmetries and Asymmetries in Evidential Support". Philosophical Studies 107: 129-142.

Fitelson, B. 2007. "Likelihoodism, Bayesianism, and Relational Confirmation". Synthese 156: 473-489. . 2011. "Contrastive Bayesianism". To appear in Contrastivism in Philosophy, M. Blaauw (ed.). New York: Routledge.

Good, I.J. 1984. Good Thinking. Minneapolis: University of Minnesota Press.

Joyce, J. 2008. “Bayes' Theorem”. In The Stanford Encyclopedia of Philosophy (Fall 2008), Edward N. Zalta (ed.). URL = http://plato.stanford.edu/archives/fall2008/entries/bayes-theorem/. Miller, D. 1994. Critical Rationalism: A Restatement and Defence. Chicago, IL: Open Court. Popper, K. 1954. "Degree of Confirmation". The British Journal for the Philosophy of Science. 5: 143-149.

Royall, R. 1997. Statistical Evidence: A Likelihood Paradigm. New York: Chapman \& Hall. Sober, E. 2008. Evidence and Evolution. Cambridge, UK: Cambridge University Press. Tentori, K., Crupi, V., Bonini, N., and Osherson, D. 2007. "Comparison of confirmation measures". Cognition 103: 107-119. 